\title{
Improved Interpretation of Satellite Altimeter Data Using Genetic Algorithms
}

\author{
Kenneth Messa \\ Department of Mathematical Sciences \\ Loyola University \\ New Orleans, LA

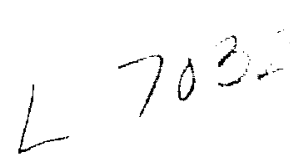 \\ (504)865-3340 \\ fax: (504)865-3347 \\ Matthew Lybanon \\ Naval Oceanographic and Atmospheric Research Laboratory \\ Stennis Space Center, MS \\ (601)688-5263
}

\section{ABSTRACT}

Gerietic algorithms (GA) are optimization techniques that are based on the mechanics of evolution and natural selection. They take advantage of the power of cumulative selection, in which successive incremental improvements in a solution structure become the basis for continued development. A GA is an iterative procedure that maintains a "population" of "organisms" (candidate solutions). Through successive "generations" (iterations) the population as a whole improves in a simulation of Darwinism's "survival of the fittest". GAs have been shown to be successful where noise significantly reduces the ability of other search techniques to work effectively.

Satellite altimetry provides useful information about oceanographic phenomena. It provides rapid global coverage of the oceans and is not as severely hampered by cloud cover as infrared imagery. Despite these and other benefits, several factors lead to significant difficulty in interpretation.

The GA approach to the improved interpretation of satellite data involves the representation of the ocean surface model as a string of parameters or coefficients from the model. The GA searches in parallel a population of such representations (organisms) to obtain the individual that is best suited to "survive", that is, the fittest as measured with respect to some "fitness" function. The fittest organism is the one that best represents the ocean surface model with respect to the altimeter data.

\section{INTRODUCTION}

Much useful information about oceanographic phenomena can be obtained from an altimeter borne on a satellite. In addition to providing rapid global coverage of the oceans, satellite altimetry bypasses other (in-situ) measurement problems. It is not as severely hampered by cloud cover as infrared imagery, and it also measures oceanographic phenomena that have no surface thermal expression. Despite the benefits of altimetry, several factors lead to significant difficulty in interpretation. Among these are atmospheric noise (from water vapor, ionospheric electrons, solar activity, and so forth), scale errors (the magnitudes of many of the errors are greater than the phenomena measured), some measurements are time dependent while other related ones are time independent (the presence of the mean dynamic topography in the reference surface or "geoid", for example) and in the calculations of the geoid itself.

In this paper we first present some background on the use of satellite altimetry data to measure the sea surface. The interpretation of these measurements is complicated by the difficulties referred to above. In order to improve our 
interpretation of the altimeter data, we turned to a technique based on an optimization procedure believed to operate effectively in nature. Known as "genetic algorithms" (GA), these techniques have been shown to be successful in many environments. Because they search in parallel a large portion of the solution space, they are able to distinguish local optima from global ones. GAs can successfully search where noise significantly reduces the ability of other search techniques to work effectively. We demonstrate the effectiveness of GAs to fit a model of the sea-surface height to data obtained from satellite altimetry.

\section{BACKGROUND}

A satellite-borne radar altimeter measures the distance from its antenna's electrical center to the instantaneous sea surface, averaged over the footprint. Sea level is the difference between the altimeter-measured distance and the satellite's height; the latter is determined independently by tracking and orbit determination. Then, the difference between sea level and the geoid, the sea surface height (SSH) residual, provides information on ocean dynamics.

The geoid is a graviational equipotential surface. The marine geoid is the shape that would be taken by a resting ocean. Since the geoid does not change, and since the oceanographic component of sea-surface variations is generally relatively small and does change with time, the long-term temporal mean of sea level is a good approximation to the marine geoid.

The situation is different when one tries to use the altimeter to measure ocean circulation. Then the "signal" is the small SSH residual that remains after the geoid is subtracted from sea level, and the "noise" is any error in knowledge of the geoid. For practical reasons, a large part of the information that goes into "geoids" comes from altimeter measurements themselves. When there are permanent oceanographic features such as the Gulf Stream, there is a significant time-independent "dynamic height" component. Being independent, this component cannot be distinguished from the true geoid without additional information. Subtraction of a geoid containing this term from the altimeter-determined sea level may introduce a serious error (Lybanon et al., 1990).

One model for the sea surface height is realized as the difference between the expected dynamic height component and the reference surface error. This model can be tested using GEOSAT altimeter measurements of the Gulf Stream region, which has a strong mesoscale signal (Calman, 1987). The following model has been proposed by Lybanon et al. (1990):

$$
\text { SSH }=A \tanh [B(X-D-E)]-F \tanh [C(X-E)]-G
$$

where $\mathrm{X}$ is the along-track coordinate of the satellite. The first term represents the instantaneous Gulf Stream, the second represents the mean Gulf Stream, and the third term is an overall bias due to orbit error or possibly other errors. The coefficients in each hyperbolic tangent represent the amplitude, the steepness of the sloping part, and the position of the curve, respectively. By fitting this model to the altimeter data, one can add the modeled mean Gulf Stream profile to the instantaneous sea surface height to allow a better description of the Gulf Stream. The key to this proposed technique is finding the coefficients from Equation (1) that best fit the altimeter data. Lybanon et al. (1988) have attempted to use standard mathematical curvefitting schemes, but have achieved only mixed success. We propose using GAs to aid this process.

\section{GENETIC ALGORITHMS}

Genetic algorithms are optimization techniques that are based on the mechanics of evolution and natural selection. In contrast to other methods that rely on a point-to-point search of the domain space, GAs use a large sample of points from the domain. Each point, called an "organism", is a candidate solution of the problem in question. The large sample of candidate solutions (called a "population") is modified through successive iterations. Each modification is based on ideas taken from Darwinian natural selection. Although randomness is a part of the process, each modification is guided by the candidate solutions that are most successful. These "fittest" organisms 
contribute the most to succeeding iterations in a simulation of "survival of the fittest". Each successive population is called a "generation". Thus we have an intitial generation, $G(0)$, and for each generation $G(t)$, the GA forms a new one, $G(t+1)$. An algorithm to implement GAs is given by:

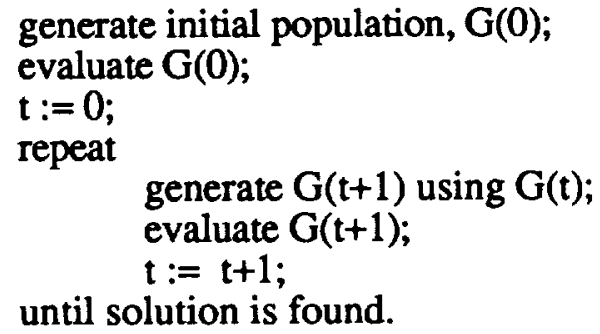

Like all generate and test methods, the GA requires the two main steps of generation and evaluation. In order to evaluate a generation, a fitness function is needed. In nature, a species responds in some way to environmental pressure. The GA analog to this pressure is the fitness function. It is built from domain specific information and returns the relative merit or fitness of the organism (Goldberg, 1989).

\subsection{Representation}

Our problem entails finding coefficients A, B, ..., $\mathrm{G}$ which yield the best fit of the altimeter data when used in Equation (1). The measurement of the goodness of fit with respect to the data $\mathbf{D}$ is the "fitness function". Since we are searching for real number values for the coefficients $A, B, \ldots, G$, the organisms for the GA used here are vectors $r=\left\langle r_{A}, r_{B}\right.$, $\ldots, r_{G}>$ of real numbers. The fitness of such an organism is the degree to which the model equation $S S H(r)$ successfully fits the data.

This view of the representation is useful at the higher level of the curve-fitting problem. However, the genetic algorithm works at a lower level-the level of bits. In order to successfully use the GA, we need to consider a representation of the real numbers $r_{i}$ at the bit level. Given upper and lower bounds for each $r_{i}, u_{i}$ and $l_{i}$ respectively, we can look at $r_{i}$ as an unsigned binary integer with $\mathrm{m}$ bits and calculate its value with respect to $l_{i}$ and $u_{i}$.

Given $a$ binary integer $b$ where $b$ is in $[0,2 \mathrm{k}-1]$, we can derive its corresponding real value using the formula

$$
r=b / 2 m *(u-1)+1
$$

where $u$ and $l$ are the upper and lower bounds respectively. Combining these two levels, we construct an organism as $\theta=\left\langle b_{A 1} b_{A 2} \ldots\right.$ $b_{A m}, b_{B 1} b_{B 2} \ldots b_{B m}, \ldots, b_{G 1} b_{G 2} \ldots b_{G m}>$ where each binary integer $b_{i 1} \quad b_{i 2} \quad \ldots b_{i m}$ corresponds to a real number $r_{i}$ which lies in the interval $\left[l_{i}, u_{i}\right]$ for $i=A, B, \ldots G$. The correspondence is given in (2).

Computing the value of the fitness function of an organism $\theta$ requires two steps: first, converting each binary integer $b_{i 1} b_{i 2} \quad \ldots b_{i m}$ into its corresponding real value $r_{i}$; then, second, evaluating the curve $\operatorname{SSH}\left(\mathrm{r}_{\mathrm{A}}, \mathrm{r}_{\mathrm{B}}, \ldots\right.$, $r_{G}$ ) at the data points of $D$.

\subsection{Evaluation}

Since the fitness function is a measurement of how well the organism fits the data, it focuses the GA toward the solution. The fitness function used here is modeled after least squares/regression. An organism $\theta$ is converted into a vector of real numbers, $r=$ $\left\langle r_{A}, r_{B}, \ldots, r_{G}\right\rangle$ using equation (2). The fitness is then computed as the sum of the squares of the differences between the $\operatorname{SSH}\left(r ; x_{i}\right)$ and $y_{i}$ (that is, the residuals). Thus,

$$
\text { fitness }(\theta)=\Sigma\left(\operatorname{SSH}\left(r ; x_{i}\right)-y_{i}\right)^{2}
$$

where the summation is a taken over all data points $\left(x_{i}, y_{i}\right)$ of $\mathbf{D}$. With this fitness function, a value of 0 is considered a perfect fit and an organism is highly fit if its fitness value is low. 


\subsection{Convergence}

The GA is designed to improve the relative merit of the population over time. While the average fitness of one generation may be lower than the preceeding one, or while the best solution from one generation may not be as good as the best from a previous one, in general, fitnesses improve as generations unfold. Figure 1 in the appendix is an example of this point.

In earlier generations, there is a great deal of variability among the organisms in a single generation. There is a wide range of fitness values in these earlier generations. As happens occasionally, a few organisms are generated whose fitnesses are exceedingly poor. This reduces the average fitness of the overall generation. The number of poor solutions generated is in proportion to the fitness of the generation as a whole. Thus, in the early generations, a larger number of poor solutions are formed. However, as the generations improve and larger numbers of the organisms have good fitnesses, the occasional poor performer does not affect the population as much. The effect of these less fit organisms in later generations is minimal.

As the generations improve, the average fitness stabilizes. As a result, most of the organisms are nearly identical. This stabilization is called "convergence", and the GA is said to converge to the organism that appears most often. Of course, at convergence, nearly all organisms are identical. This commonality is the solution to the problem.

\subsection{Operators}

GAs are based on many of the same principles as those found in natural selection. They employ several operators and principles that are generally derived form those that occur in nature. There are three principal operators at work in GAs: selection, crossover and mutation. The first of these, selection, is the analog of the principle in natural selection that organisms that are most fit are most favorably disposed for participation in mating, thereby passing their genetic information to their offspring. The selection operator chooses individuals from the population so that those with high fitnesses have greater probability of being selected. This focus toward the highly fit individuals is what drives genetic algorithms.

The method of selection that was used here is stochastic sampling without replacement, called "expected value" by Goldberg (1989). In addition, we have used de Jong's (de Jong 1975) "elitist" strategy, whereby the single best organism form one generation is placed unchanged into the next generation. This strategy gives a little more weight to the best organism than might be achieved from selection alone and prevents the possibility that the best organism might be lost early through crossover or mutation.

The GA analogy to mating is called crossover. The crossover operator provides a mixing of the genetic material from the parents, and globally, it mixes the genetic information of the whole population. It is the mixing of the "genes", the stirring of the pot of genetic material, that gives the GA robustness. The two organisms chosen by selection are combined to form a new individual with similarities to both parents. If the mixing is done carefully, a large amount of genetic material can be tested. Although selection focuses the genetic algorithm, it is crossover that adds variety.

We employed a "two-point" crossover. Twopoint crossover proceeds as follows: Once the organisms (the "parents") have been selected for mating, two bit positions are chosen at random. The middle segments between these bit positions of the two organisms are interchanged to form two new organisms. These new organisms (the "offspring") are added to the next generation. The process of selection and crossover is repeated until the new generation has the same number of members as the previous generation.

While selection and crossover are the chief operators used in GAs, there are numerous other minor operators proposed to strengthen GAs under certain circumstances. It has been shown that for certain applications, these minor operators can add to the GA's efficiency or prevent it from converging to a local optimum rather than global one. For example, it sometimes happens that the GA converges to a 
solution prematurely. This is due to the fact that crossover only mixes the genetic material that is present in the initial population; it doesn't introduce any new material. In nature, new genes are introduced into a species through mutation. Analogously in GAs, a mutation operator is used to modify an organism occasionally in order to add new genetic material into the population and to prevent premature convergence.

We used a mutation method that adds a real value $\varepsilon$ to (or subtracts it from) the organism's value at one of the coefficents. We kept the probability of a mutation low. Thus, if by chance a particuplar organism was to be mutated at one of its coefficients, $r$, then a small $\varepsilon_{\mathrm{i}}$ was added to (or subtracted from) $r$. The value of $\varepsilon$ is a power of 2 ranging from 1

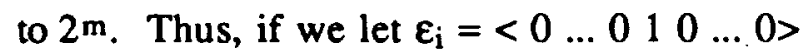
with 1 in the i-th position and 0's elsewhere, then this mutation method effectively adds $\varepsilon_{\mathrm{i}}$ to (or subtracts $\varepsilon_{i}$ from) the coefficient of the organism to be mutated.

\section{RESULTS}

The GA technique outlined above is dependent on the choice of boundary points $l_{i}$ and $r_{i}$ for each $\mathrm{i}=\mathrm{A}, \mathrm{B}, \ldots$, G. Knowledge of the problem domain may by useful to ascertain these boundaries. If the knowledge is inadequate, a degree of experimentation may be required. Our knowledge of the problem, gained in part by previous work (Lybanon et al., 1988), gave us some information about the coefficients. We knew that the amplitudes of the curves (coefficients $A$ and $F$ ) were positive and less than 1. Likewise we knew the slope coefficients ( $B$ and $C$ ) were also positive and less than 1. Hence, for all these values, we used domain intervals $[0,1]$. The error of $G$ was small, but its sign was unknown to us. We used $[-1,1]$ as its domain interval. The difficult values to determine were the position coefficients D and E.

We began with intervals of length 100 for each of $D$ and $E$. We were prepared to test several intervals of this length, $[-50,50],[-75,25]$,
$[-25,75]$, and so forth. If necessary, we might have had to increase the length of the intervals to 200 or more. In any case, we were prepared to do the experimentation needed for the GA. The results given below show that we needed only the second interval mentioned.

After several runs using the domain interval $[-50,50]$ for coefficents $D$ and $E$, it became clear that this domain did not include the value for $E$, and perhaps not $D$ either. One run placed the optimal E exactly at -50 , indicating either a coincidence that we chose an endpoint of the domain interval very near the optimal value or the possibility that E lay below -50 . The other runs had somewhat low errors, yet the values of the coefficients were not near each other. This could also mean that we stopped the GA too soon, before it had a chance to converge. See Table 1 . On the strength of the former observation, we abandoned the $[-50$, $50]$ interval for $E$ in favor of $[-75,25]$. If indeed -50 was the optimal value for $E$, this new interval would bear this out. If the error was due to the optimal $\mathrm{E}$ being smaller than -50 , then this or another interval would be better. If the results for the new interval were likewise inconsistent, we would allow the GA to run for several generations longer and compare results. Since D did not seem to suffer the same error as $E$, we were not quick to adjust D's domain interval. Table 2 exhibits the results obtained with these new intervals. The value of $E$ derived here supports our earlier decision to reduce the lower bound for E's interval.

\begin{tabular}{|c|c|c|c|}
\hline coefficient & run \# 1 & run \# 2 & run \# 3 \\
\hline A & .273373 & .191273 & .240241 \\
\hline B & .435305 & .499703 & .504699 \\
\hline C & .058454 & .008573 & .008887 \\
\hline $\mathrm{D}$ & 15.7059 & -46.5097 & -40.3814 \\
\hline $\mathrm{E}$ & -50.0000 & 13.6979 & 7.37317 \\
\hline $\mathrm{F}$ & .260792 & .640602 & .851496 \\
\hline $\begin{array}{r}G \\
\text { error }\end{array}$ & $\begin{array}{l}-.028586 \\
.612744\end{array}$ & $\begin{array}{l}-.352537 \\
.903165\end{array}$ & $\begin{array}{l}-.414537 \\
.825254\end{array}$ \\
\hline
\end{tabular}


Table 2. Coefficients obtained with intervals $[-75,25]$ for $E$ and $[-50,50]$ for $D$.

$\begin{array}{crrr}\text { coefficient } & \text { run \# 1 } & \text { run \# 2 } & \text { run \# 3 } \\ \text { A } & .186314 & .186297 & .186302 \\ \text { B } & .757704 & .757711 & .757642 \\ \text { C } & .174833 & .174889 & .174872 \\ \text { D } & 24.7986 & 24.8003 & 24.7997 \\ \text { E } & -58.7977 & -58.7995 & -58.7989 \\ \text { F } & .162858 & .162844 & .162848 \\ \text { G } & -.025868 & -.025869 & -.025868 \\ \text { error } & .558747 & .558747 & .558747\end{array}$

To contrast these results, we tried the interval $[-25,75]$. We obtained inconsistencies in all runs, as might be expected, as the correct value of $E$ was far removed from this interval.

In this problem, we had some knowledge of the coefficients. However, one can obtain very accurate results with very little knowledge, if one is allowed to experiment. Beginning with a domain interval of $[-100,100]$ for each coefficent, after several runs we were able to revise our results for $A, F$, and $G$ as lying in the interval $[-5,5]$. The experiments gave us no information about $B, C, D$, or $E$, however. We ran the GA several more times on the revised intervals. At this point we were able to further narrow the intervals for some of the coefficients. By repeating this process through just four stages of interval reduction, we were able to obtain the results in Table 3.

When small enough intervals are used (gained through either experimentation or knowledge of the model), one can get a very accurate fit of the data. With an accurate fit, the dynamic height component can be removed yielding a more accurate interpretation of the data. See Figure 2 in the appendix. It shows the original altimeter data of the Gulf Stream and the adjusted values after the dynamic component has been removed.

\section{SUMMARY}

We have demonstrated that genetic algorithms can be used successfully to improve the interpretation of altimeter data in a model for the sea surface height. There are several strengths to this approach. First, it does not
Table 3. Coefficients obtained after several stages of reducing the intervals

$\begin{array}{crrr}\text { coefficient } & \text { run \# 1 } & \text { run \# 2 } & \text { run \# 3 } \\ \text { A } & .186290 & .186290 & .186290 \\ \text { B } & .757685 & .757680 & .757682 \\ \text { C } & .174907 & .174907 & .174907 \\ \text { D } & 24.8009 & 24.8009 & 24.8009 \\ \text { E } & -58.8000 & -58.8000 & -58.8000 \\ \text { F } & .162838 & .162838 & .162838 \\ \text { G } & -.025870 & -.025870 & -.025870 \\ \text { error } & .558747 & .558747 & .558747\end{array}$

require complex calculation nor is it difficult to set up. Second, it is accurate in its present form. With 32 bit representation of integers, we easily obtained 4,5 , or 6 significant digits. More accuracy can be achieved with minor revisions. Finally, the results were consistent, although the initial genetic algorithm parameters needed to be established at the beginning.

The method suffers from some weaknesses, however. First, the initial set of parameters is not univerally known. There must be some experimentation on these parameters initially and more experimentation on these if there is a significant change in the structure of the model. Second, the method requires some knowledge of the model coefficients. This knowledge can be gained through inspection of the data or of the function itself, or it can be gained through experimentation. In either case, the genetic algorithm method is a viable technique for improving the interpretation of the altimetry data used in this model.

\section{ACKNOWLEDGEMENTS}

This research was sponsored by the U. S. Navy Office of Naval Technology, CDR Lee Bounds, Program Manager, and by the Space and Naval Warfare Systems Command, LCDR William Cook, Program Manager.

Kenneth Messa's work was partially supported by the Naval Oceanographic and Atmospheric Research Laboratory through the U. S. Navy/ASEE Summer Faculty Research Program. 
This is NOARL Contribution Number PR 91:121:321. It is approved for public release; distribution is unlimited

\section{REFERENCES}

Baker, J. E. (1987). Reducing bias and inefficiency in the selection algorithm. Genetic Algorithms and Their Applications: Proceedings of the Second International Conference on Genetic Algorithms, 14-21.

Calman, J. (1987). Introduction to sea-surface topography from satellite altimetry. Johns Hopkins APL Technical Digest 8(2), 206-211.

Daniel, C. \& Wood, F. S. (1980). Fitting Equations to Data, 2nd Ed. New York: John Wiley \& Sons.

De Jong, K. A. (1975). An analysis of the behavior of a class of genetic adaptive systems. (Doctoral dissertation, University of Michigan). Dissertation Abstracts International 36(10), 5104B.

Goldberg, D. (1989). Genetic Algorithms in Search, Optimization, and Machine Learning. Reading, MA: Addison-Wesley.

Grefenstette, J. (1986). Optimization of control paramenters for genetic algorithms. IEEE Transactions on Systems, Man and Cybernetics, Vol. SMC-16(1), Jan/Feb 1986.

Guest, P. G. (1961). Numerical Methods of Curve Fitting. Bristol, Great Britain: Cambridge University Press.

Holland, J. (1975). Adaption in Natural and Artificial Systems, Ann Arbor, Michigan: University of Michigan Press.

Lybanon, M. \& Messa, K. (1991). Genetic Algorithm Simulation to Improve Altimetric Sea-Surface Height Residuals, to appear in Proceedings of SimTec ' 91 .
Lybanon, M., Crout, R., Johnson, C. \& Pistek, P. (1990). Operational altimeter-derived oceanographic information: The NORDA GEOSAT ocean applications program, Journal of Atmospheric and Oceanic Technology, 7(3), 357-376.

Lybanon, M., Johnson, D. R. \& Romalewski, R. S. (1988). Separation of the mean Gulf Stream topography from an altimeter-derived reference surface, $E O S$ Transactions, American Geophysical Union, (69) 44, 1281.

Lybanon, M. \& Crout, R. L. (1987). The NORDA GEOSAT ocean applications program, Johns Hopkins APL Technical Digest, (8)2.

Suh, J. Y. \& Van Gucht, D. (1987). Incorporating information into genetic search. Genetic Algorithms and Their Applications: Proceedings of the Second International Conference on Genetic Algorithms, 100-107. 


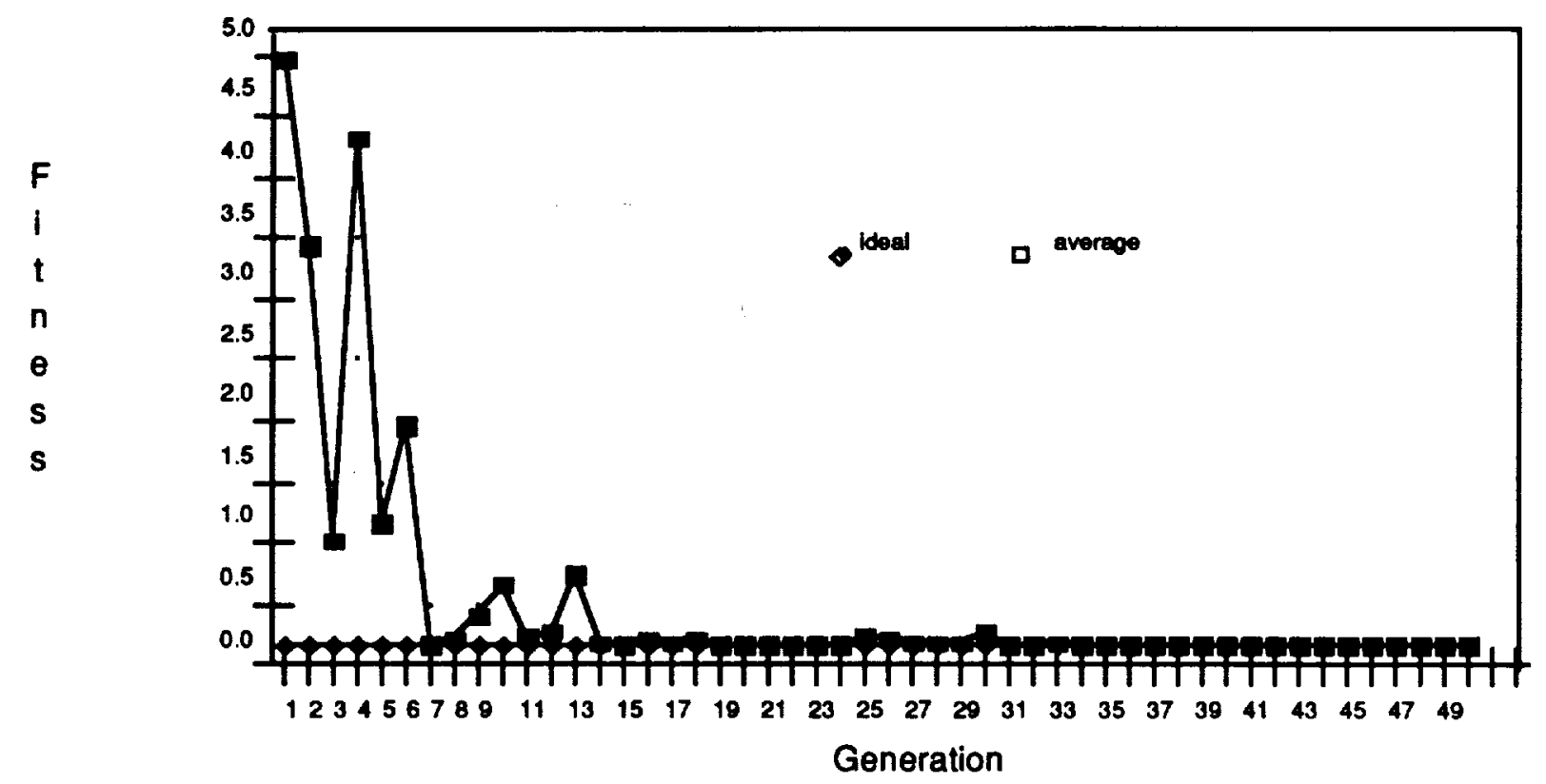

Figure 1 Average fitness of a population demonstrating convergence in a Genetic Algorithm

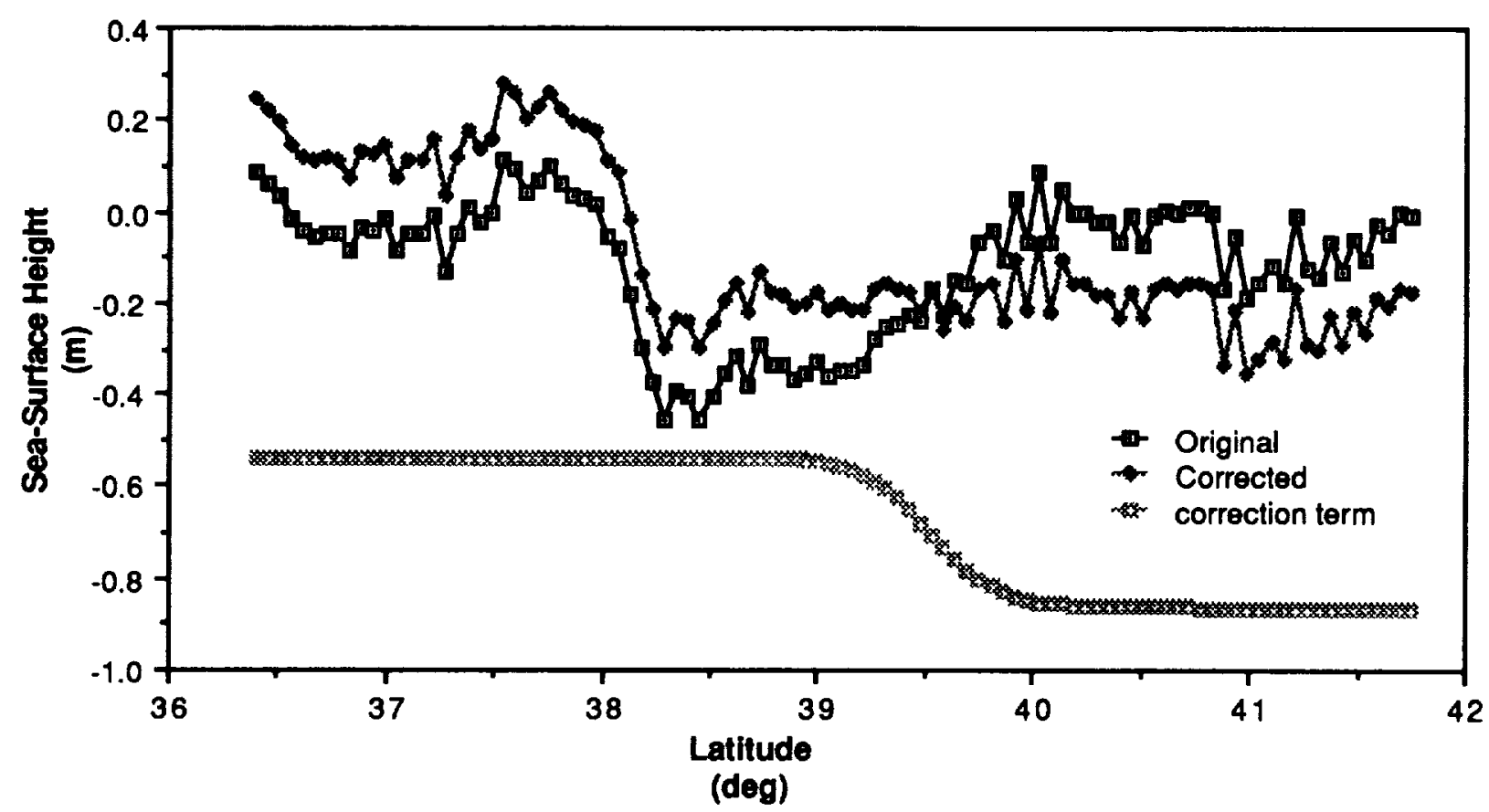

Figure 2. Original altimeter data and Correction. Correction term has been displaced for easier viewing. 